\title{
A Case of Solitary Metastasis from a Hepatocellular Carcinoma to the Sacral Bone Successfully Treated with Percutaneous Cryoablation
}

\author{
1) Department of Radiology, Okayama University Hospital, Okayama, Japan \\ 2) Center for Innovative Clinical Medicine, Okayama University Hospital, Okayama, Japan \\ 3) Department of Proton Beam Therapy, Okayama University Graduate School of Medicine, \\ Dentistry and Pharmaceutical Sciences, Okayama, Japan \\ 4) Department of Hepatology, Kagawa Prefectural Central Hospital, Takamatsu, Japan
}

Noriyuki Umakoshi ${ }^{1)}$, Hideo Gobara ${ }^{1)}$, Takao Hiraki ${ }^{1)}$, Toshihiro Iguchi ${ }^{1)}$, Hiroyasu Fujiwara ${ }^{1)}$, Jun Sakurai', Yusuke Matsui $^{1)}$, Yoshihisa Masaoka ${ }^{1)}$, Hiroki Ihara ${ }^{3)}$, Toshiaki Wada ${ }^{1)}$, Tomonori Seno ${ }^{4)}$, Susumu Kanazawa ${ }^{1)}$

\begin{abstract}
A 70-year-old woman, who had previously undergone a hepatectomy for hepatocellular carcinoma (HCC), developed a solitary metastasis in the sacral bone with the symptom of limb pain. The tumor had been in remission for 2 years, after radiotherapy and chemotherapy with sorafenib; however, it recurred locally. Although we performed transcatheter arterial embolization (TAE) on the patient, her limb pain worsened gradually. We treated the patient further with percutaneous cryoablation. On the day after she underwent cryoablation, the patient developed a disturbance in gait with a dorsiflexion disorder of the left ankle. These symptoms were believed to be caused by a cryoinjury to the L5 nerve root, which was adjacent to the sacral tumor. After rehabilitation training for several days, the patient became ambulatory with an ankle brace. At her most recent follow-up visit, 18 months after the treatment, the patient did not exhibit the presence of either local tumor progression nor new metastases.
\end{abstract}

Key words: percutaneous cryoablation, transcatheter arterial embolization, hepatocellular carcinoma, bone metastasis

(Interventional Radiology 2017; 2: 14-18)

\section{Introduction}

The bones are one of the most common extrahepatic sites of metastases from hepatocellular carcinomas (HCC) [1-5]. Bone metastases typically occur in multiple locations; therefore, these are usually treated palliatively, by radiotherapy and chemotherapy with sorafenib $[1,6]$. However, recent studies have shown that more aggressive local treatments are effective in select patients who have either solitary or few bone metastases [2-5]. Therapeutic options for such patients include surgical resections [2], transcatheter arterial emboli- zations (TAEs) [3], thermal ablations, such as, radiofrequency ablations (RFA) [4], and cryoablations [5]. We present a case of solitary metastasis from an HCC to the sacral bone that was successfully treated with percutaneous cryoablation.

\section{Case Report}

A 70-year-old woman, who had previously undergone a hepatectomy for HCC 4 years ago, developed a solitary metastasis of her tumor in the sacral bone with symptoms of limb pain. She had previously received radiotherapy (dose, 

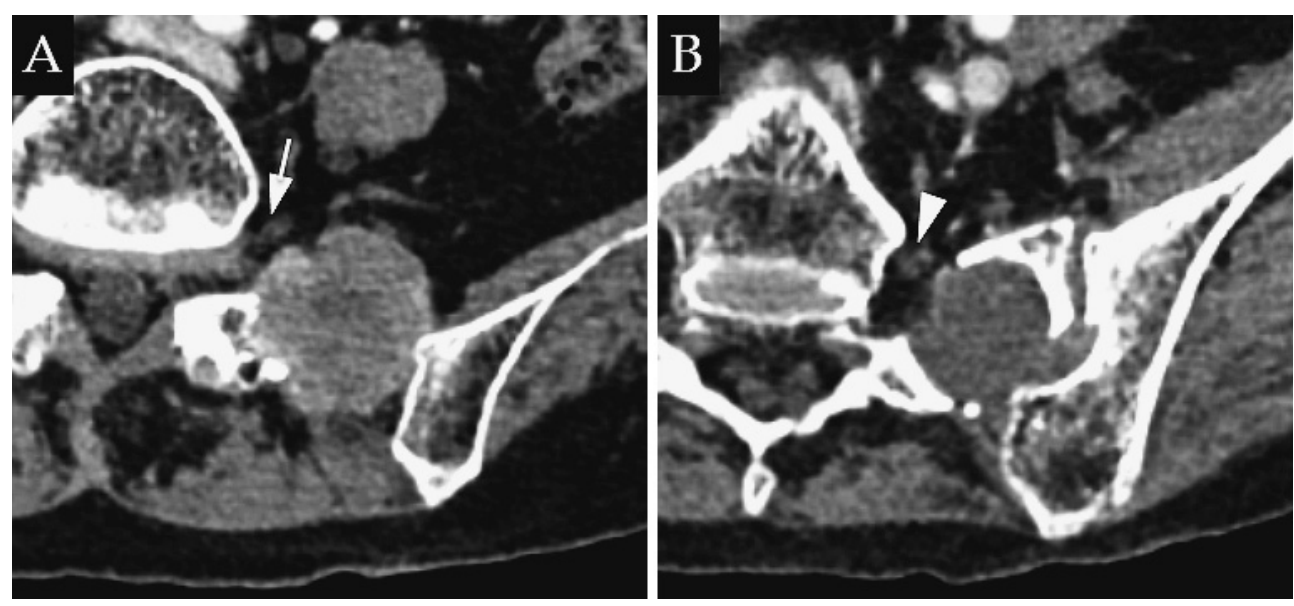

Fig. 1. Contrast-enhanced computed tomography images show the presence of an osteolytic bone lesion on the left side of the sacral bone (A) the $\mathrm{L} 5$ nerve root (indicated by the arrow) level; (B) the S1 nerve root (indicated by the arrowhead) level.

42 Gy, 14 fractions) followed by chemotherapy with sorafenib (dose, $400 \mathrm{mg} /$ day) for her HCC. Although the patient had been in remission for 2 years after this treatment, the tumor recurred locally in her sacral bone. Additionally, she displayed elevated blood levels of alpha-fetoprotein (AFP). The patient was referred to our hospital for the treatment of the recurred tumor. Computed tomography (CT) and magnetic resonance imaging (MRI) scans demonstrated the presence of a 5-cm osteolytic bone lesion on the left side of the sacral bone; the L5 and S1 nerve roots were located anterior to the tumor (Fig. 1A, B).

Initially, we performed TAE for treatment of the bone metastasis. Angiography showed the presence of neovascularity and tumor stain on the sacral tumor from multiple feeding arteries, including the left lateral sacral artery, left iliolumbar artery, and left fourth lumbar artery. TAE was performed using a 1-mm sized gelatin sponge particle $\left(\right.$ Gelpart $^{\circledR}$, Nippon Kayaku, Tokyo, Japan) without ethiodized oil or anti-cancer drugs. Dynamic MRI scans obtained one day after the patient underwent TAE showed significantly reduced contrast enhancement of the tumor, but thin, residual enhancement persisted at the tumor periphery. The blood levels of AFP in the patient decreased temporarily, but were re-elevated 2 months after undergoing TAE. Additionally, the symptom of limb pain experienced by the patient worsened.

Among the available ablation modalities, cryoablation was regarded as the optimal treatment option, mainly because of the visibility of the ablation zone during the procedure (i.e. iceball). After obtaining approval from the institutional review board and written informed consent from the patient, we treated the metastasis with cryoablation using an argonhelium based system (Cryo-Hit; Galil Medical Yokneam, Israel). Because the tumor was large in size $(5 \mathrm{~cm})$ and was present adjacent to the L5 and S1 nerve roots, we divided it into three parts (i.e. the upper, middle, and lower parts in a coronal image), to ensure the safe and effective treatment of the entire tumor (Fig. 2A). We performed ablation on the lower part of the tumor, using CT fluoroscopic guidance.
Three 17-gauge cryoprobes (Ice-Seed; Galil Medical) were placed in a triangular shape, and the tumor was ablated in two freeze cycles of 10 minutes each, which were separated by 2 minutes of passive thawing.

During the freezing process, intermittent monitoring was performed using CT fluoroscopy. In addition, a conventional CT scan was obtained immediately after each freeze cycle (Fig. 2a, b, c). During the ablations of the lower and middle parts of the tumor, the S1 nerve root was not included in the low-density iceball and, thus, was successfully preserved. However, during the ablation of the upper part of the tumor, CT images taken after the second freeze cycle showed that the low-density iceball involved the entire L5 nerve root (Fig. 3A, B). The patient did not complain of pain during or immediately after the ablation procedure.

On the day after she underwent cryoablation, the patient developed a disturbance in gait with a dorsiflexion disorder of the left ankle joint. These symptoms were believed to be caused by cryoinjury of the L5 nerve root, which was adjacent to the sacral tumor. After undergoing rehabilitation training for several days, the patient became ambulatory with the aid of an ankle brace. No enhancement of the ablation zone was observed on MRI scans obtained one day after performing the cryoablation procedure (Fig. 4). The blood levels of AFP in the patient decreased immediately after the treatment, and remained unchanged during the follow-up of the patient for 18 months (Fig. 5). At her most recent follow-up visit at 18 months after the treatment, the patient did not show either local tumor progression nor new metastases, although her ankle symptoms have not improved.

\section{Discussion}

Both radiotherapy and chemotherapy with sorafenib are standard treatments for bone metastases from HCCs $[1,6]$. These treatments had previously been applied in the current case, and had been effective for 2 years. When cancers recur 


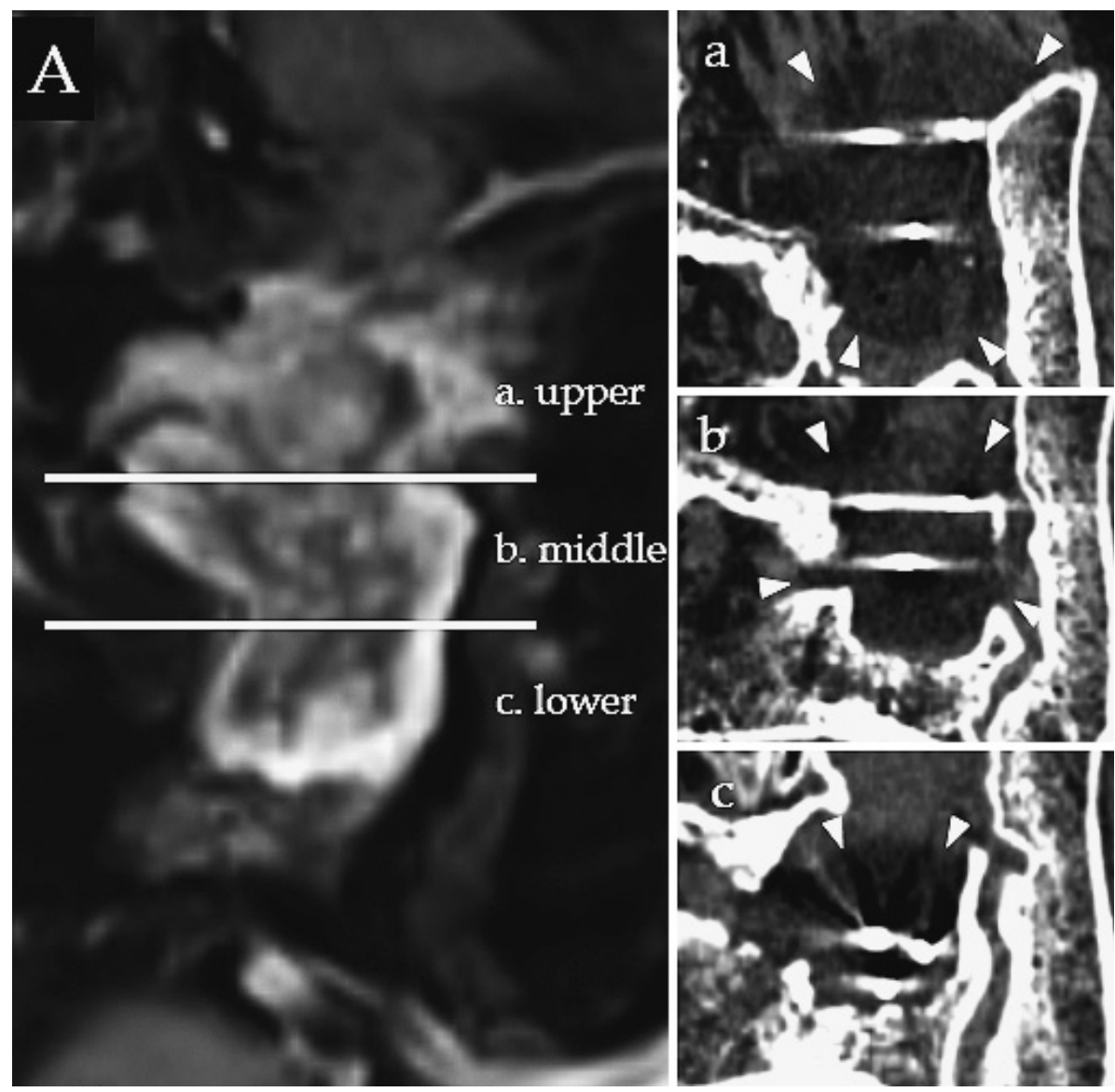

Fig. 2. (A) Dynamic magnetic resonance imaging scans. The osteolytic bone lesion was divided into three parts (the upper, middle, and lower parts in a coronal image). Computed tomography scans were obtained immediately after each freeze cycle. A coronal reconstruction image shows the iceball (indicated by the arrowhead). (a) The upper, (b) middle, (c) and lower parts of the tumor.
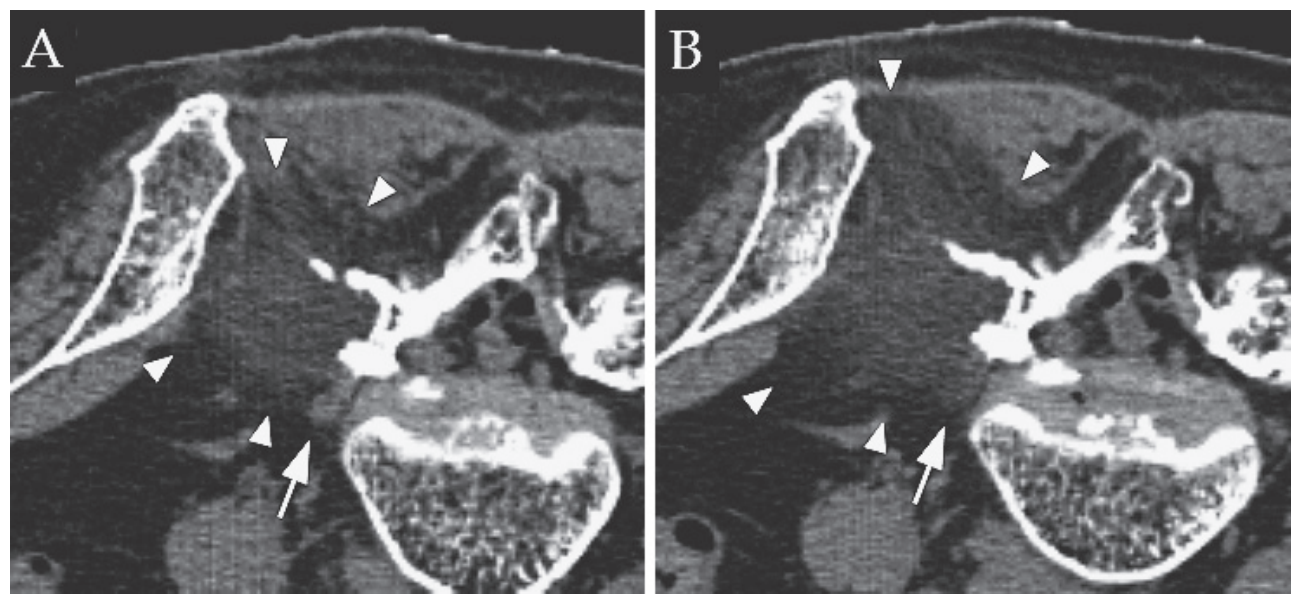

Fig. 3. Computed tomography images obtained at the L5 nerve root level after first (A) and second freezing (B). The iceball (indicated by the arrowhead) includes the entire L5 nerve root after the second freezing cycle (indicated by arrow).

after these treatments, repeat radiotherapy may be contraindicated, because metastases may be situated near the bowels or in previously irradiated tissues. Surgical resections have been shown to improve survival rates in select patients [2], and might present options for treatments in such cases. However, in the current case, percutaneous treatment was deemed more suitable because it is less invasive.

Several studies have focused on the effectiveness of treating patients with TAE for pain relief from bone metastases from HCCs $[3,7,8]$. For patients, treatments with TAE may be more advantageous than undergoing radiotherapy, because pain relief occurs sooner with the former treatment 

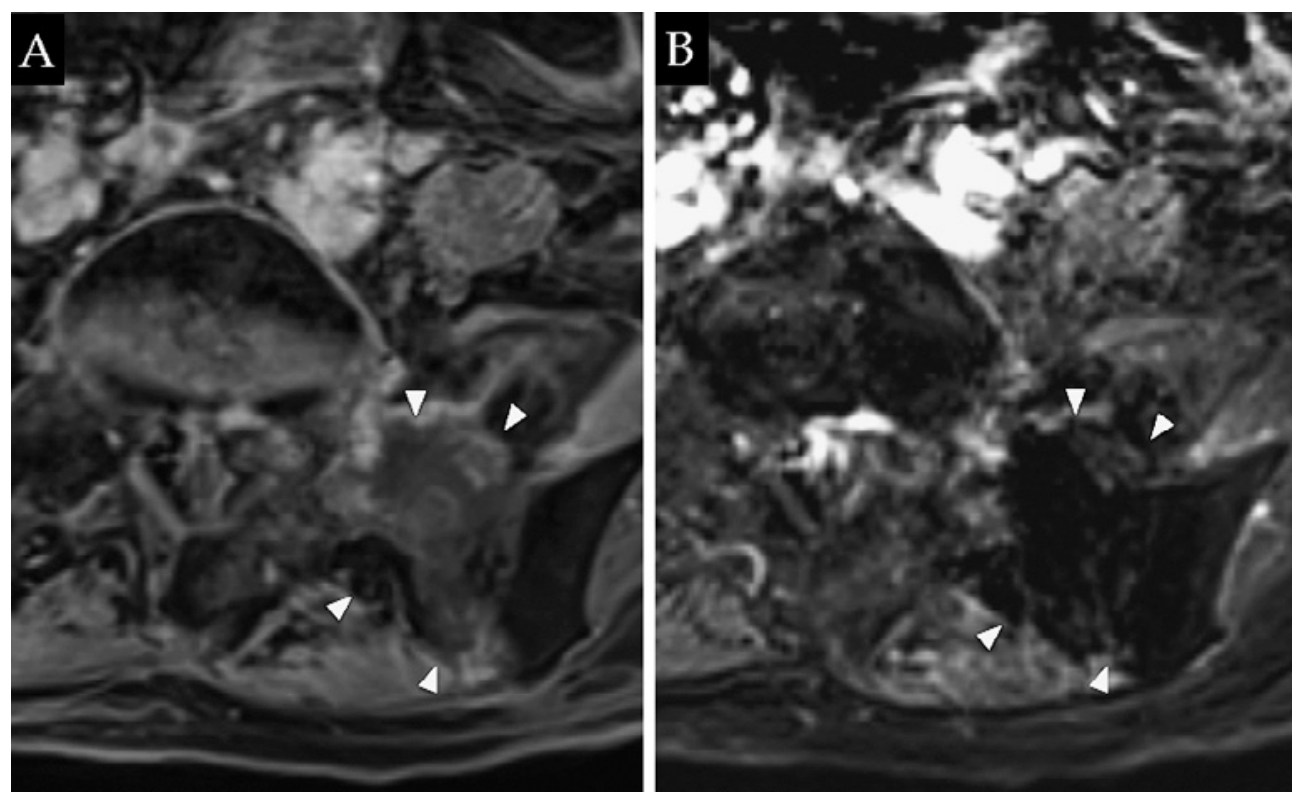

Fig. 4. Magnetic resonance imaging scans obtained one day after the patient underwent cryoablation. Images from a dynamic subtraction magnetic resonance imaging scan (A) and a subtraction scan (B) show the disappearance of contrast enhancement in the ablated zone (indicated by the arrowhead).

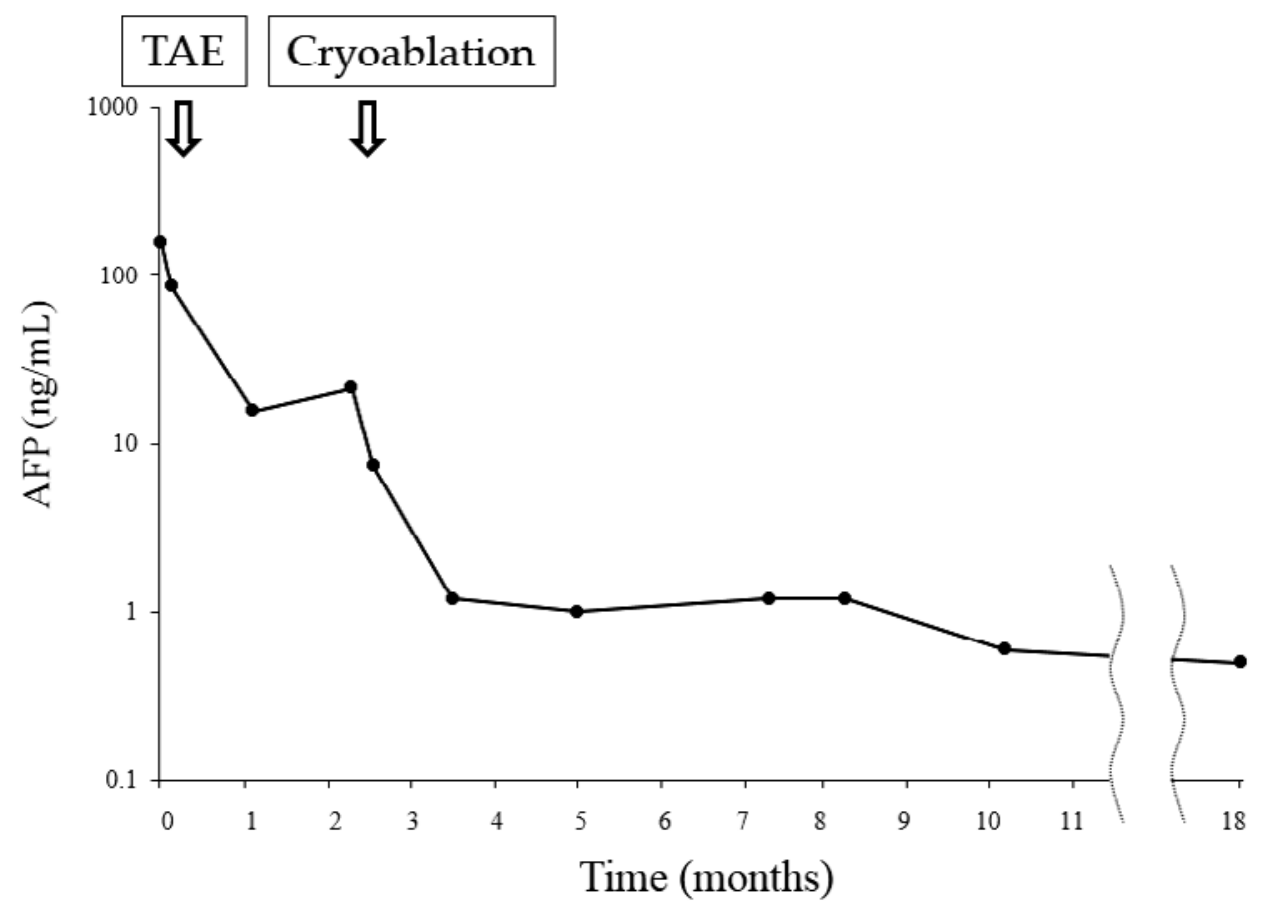

Fig. 5. Change in blood levels of tumor marker. The blood levels of alpha-fetoprotein markedly decreased after cryoablation, and remained low for 18 months thereafter. TAE: transcatheter arterial embolization

[8]. In the current case, TAE was performed; however, the tumor and the associated symptoms recurred soon after. These results suggested that it was difficult to completely treat the tumor with TAE because of the presence of multiple, complex feeding arteries.

Percutaneous cryoablation is a minimally invasive treatment procedure [5], and its feasibility and efficacy have been reported mainly in renal tumors [9]. Among the available ablation modalities, we believed that cryoablation was the most suitable treatment method for the current case, mainly because of the visibility of the ablation zone during the performance of this procedure (i.e. iceball). Although RFA is known to provide effective thermal ablations for hepatic lesions [4], in the current case, we were concerned that 
treatment with RFA might cause severe periprocedural pain in the patient.

There are a limited number of reports on the efficacies of local cryoablations for bone tumors. McMenomy et al. reported a local control rate of $87 \%$ at a median follow-up time of 21 months after performing cryoablations with curative intent, in 52 cases of musculoskeletal metastases. They included only one patient with metastases from HCC in their study, but did not achieve local control in the patient[5].

Most complications from cryoablations for musculoskeletal tumors may result from damages to surrounding structures due to freezing. The occurrence of complications is correlated with the iceball volume in the cryoablation procedure, and the rate of major complications due to cryoablations in musculoskeletal systems is reported to be 5\% [5]. In the current case, we were concerned about injuries to the adjacent nerves, and thus carefully monitored these nerves using CT fluoroscopy, while performing cryoablation. However, the artifacts induced while using metallic cryoprobes presented obstacles for detailed monitoring. Although the S1 nerve root was successfully preserved, we failed to detect the injury to the L5 nerve that occurred during the procedure. Blocking blood flow (heat-pump effect) by TAE may potentially enlarge the iceball volume more than expected, and contribute to complete tumor necrosis. Resultantly, the patient developed a disturbance in gait after undergoing the procedure. She soon became ambulatory with an ankle brace. Procedural guidance by MRI scanning may have been more appropriate in this situation [10].

In conclusion, treatment with percutaneous cryoablation in a patient with a solitary metastasis to the sacral bone achieved almost complete necrosis of the tumor, as well as, pain relief accompanied by a few tolerable complications in the patient.
Conflict of interest: The authors declare that they have no conflicts of interest concerning this manuscript.

\section{References}

1. Hayashi S, Tanaka H, Hoshi H. Palliative external-beam radiotherapy for bone metastases from hepatocellular carcinoma. World J Hepatol 2014; 6: 923-929.

2. Chan KM, Yu MC, Wu TJ, et al. Efficacy of surgical resection in management of isolated extrahepatic metastases of hepatocellular carcinoma. World J Gastroenterol 2009; 15: 5481-5488.

3. Uemura A, Fujimoto H, Yasuda S, et al. Transcatheter arterial embolization for bone metastases from hepatocellular carcinoma. Eur Radiol 2001; 11: 1457-1662.

4. Kashima M, Yamakado K, Takaki H, et al. Radiofrequency ablation for the treatment of bone metastases from hepatocellular carcinoma. AJR 2010; 194: 536-541.

5. McMenomy BP, Kurup AN, Johnson GB, et al. Percutaneous cryoablation of musculoskeletal oligometastatic disease for complete remission. J Vasc Interv Radiol 2013; 24: 207-213.

6. Bruix J, Raoul JL, Sherman M, et al. Efficacy and safety of sorafenib in patients with advanced hepatocellular carcinoma: subanalyses of a phase III trial. J Hepatol 2012; 57: 821-829.

7. Nagata $Y$, Nakano Y, Abe M, et al. Osseous metastases from hepatocellular carcinoma: embolization for pain control. Cardiovasc Intervent Radiol 1989; 12: 149-153.

8. Murakami R, Baba Y, Furusawa M, et al. Short communication: the value of embolization therapy in painful osseous metastases from hepatocellular carcinomas; comparative study with radiation therapy. Br J Radiol 1996; 69: 1042-1044.

9. Kapoor A, Wang Y, Dishan B, et al. Update on cryoablation for treatment of small renal mass: oncologic control, renal function preservation, and rate of complications. Curr Urol Rep 2014; 15 : 396.

10. Mogami T, Harada J, Kishimoto K, et al. Percutaneous MR-guided cryoablation for malignancies, with a focus on renal cell carcinoma. Int J Clin Oncol 2007; 12: 79-84. 